Editorial

\title{
Special Issue: Development and Application of Nonlinear Dissipative Device in Structural Vibration Control
}

\author{
Zheng Lu ${ }^{1, *(1)}$, Ying Zhou ${ }^{1}$, Tony Yang ${ }^{2}$ and Angeliki Papalou ${ }^{3}$ \\ 1 Research Institute of Structural Engineering and Disaster Reduction, College of Civil Engineering, \\ Tongji University, Shanghai 200092, China; yingzhou@tongji.edu.cn \\ 2 Department of Civil Engineering, University of British Columbia, Vancouver, BC V6T 1Z4, Canada; \\ yang@civil.ubc.ca \\ 3 Department of Civil Engineering, Technological Educational Institute (T.E.I.) of Western Greece, \\ 26334 Patras, Greece; papalou@teiwest.gr \\ * Correspondence: luzheng111@tongji.edu.cn; Tel.: +86-21-6598-6186
}

Received: 21 May 2018; Accepted: 22 May 2018; Published: 23 May 2018

This Special Issue (SI) of Applied Sciences on Development and Application of Nonlinear Dissipative Devices in Structural Vibration Control contains papers that focus on the development and application of innovative nonlinear dissipative systems that mitigate the potentially catastrophic effects of extreme loading by incorporating new materials or effective mechanical control technologies. Moreover, the new nonlinear analytical methods for distinctive vibrating structures under different excitations are also highlighted in this Special Issue. It is notable that many research areas, especially those related to civil engineering, have placed more importance on the nonlinear characteristics of both vibrating structures and dissipation devices. This is mainly because under strong excitations, such as severe earthquakes, the vibrating structures tend to yield and generate excessive displacement, which leads to material and geometric nonlinearities, respectively. Both of these nonlinearities have significant effects on the seismic performance of vibrating structures and the damping effectiveness of dissipative devices. Additionally, the nonlinear dampers present more advantages in energy dissipation than linear dampers, such as wide frequency bands of vibration attenuation and high robustness. Therefore, these nonlinear dampers have been utilized in many different cases. For example, nonlinear fluid viscous dampers are applied to control the large maximum bearing displacement of isolation systems; pounding tuned mass dampers are employed to alleviate the excessive vibration of power transmission towers; and self-powered magnetorheological dampers are used to suppress the undesirable vibration of long stay cables.

We have been particularly interested in receiving manuscripts that encompass the development of efficient and convenient composite nonlinear dampers; experimental investigation, advanced modeling and systematical theoretical analysis of nonlinear dynamic systems; and optimization of creative nonlinear dampers and damping mechanisms. Therefore, the papers we have received are on a wide variety of topics, which can be mainly divided into three categories: academic fundamental phase, current cutting-edge research and industrial application phase. It is worth mentioning that to broaden the scope of nonlinearity, apart from the nonlinear dissipative devices, the specific structures that contain nonlinear connections or express nonlinear behaviors and the base-isolated structures whose isolators would yield under large displacements are also the targets of this special issue. Moreover, to reinforce the point that linear dampers are capable of producing desirable damping performance under certain circumstances, the recent research pertaining to the linear dampers, including the tuned mass damper and eddy current tuned mass damper (the damping force produced by the eddy currents is proportional to the relative velocity), are also included in this special issue. 
This special issue has already published 13 very high-quality papers. The author groups represent currently active researchers in the structural vibration control area. The topics are not only current (cutting-edge research) but also of great academic (fundamental phase) and industrial (applied phase) interest. The readers will observe that compared to linear dissipative devices, the application of nonlinear dissipative devices in civil engineering is just in its preliminary stages, with most of the research concentrating on the theoretical study, numerical simulation and experimental study. Hence, more further efforts should be made on the application phase of nonlinear dampers. The papers are cited below, with brief comments for each paper concerning the main topic and contributions of the paper.

Academic fundamental phase. Due to the fact that the traditional linear/nonlinear dampers cannot meet the demands of vibration attenuation in severe conditions, such as a power transmission tower undergoing multi-component seismic excitations, a submerged pipeline being subjected to seawater environments and a building structure withstanding debris flow, some authors have subsequently proposed new linear/nonlinear dissipative devices, which has enriched the academic fundamental research of linear/nonlinear dissipative devices. The papers in this category and corresponding comments are listed below:

(1) Tian, L.; et al. Vibration Control of a Power Transmission Tower with Pounding Tuned Mass Damper under Multi-Component Seismic Excitations [1].

The very first submitted and accepted paper of this Special Issue proposes a new nonlinear dissipative device that can be applied to increase the seismic resistance of a power transmission tower. This device is namely the pounding tuned mass damper (Pounding TMD), which combines the impact damper and the tuned mass damper (TMD). The main contributions of this paper are as follows: (a) a three-dimensional finite element modal of a practical power transmission tower attached with TMD/Pounding TMD is established to verify the superior effectiveness of Pounding TMD over TMD; and (b) parametric analysis was carried out through this model, including mass ratio, ground motion intensity, gap and incident angle.

(2) Chen, J.; et al. Experimental Study on Robustness of an Eddy Current-Tuned Mass Damper [2].

In this paper, the robustness of an eddy current tuned mass damper (ECTMD) is investigated experimentally through the vibration control of a cantilever beam, with comparison of its results to the robustness of a tuned mass damper. The experimental results indicate that the damping performance of the ECTMD is superior to that of the TMD, which is mainly due to its higher robustness under both free vibration and forced vibration.

(3) Wang, W.; et al. Experimental Study on Vibration Control of a Submerged Pipeline Model by Eddy Current Tuned Mass Damper [3].

This paper utilizes an eddy current tuned mass damper to suppress the excessive vibration of submerged pipelines and validates the feasibility of eddy current damping in a seawater environment through an experimental study. The test results show that the damping provided by the eddy current in a seawater environment is only slightly varied compared to that in an air environment. Furthermore, with the optimal ECTMD control, the vibration response of the submerged pipeline is significantly decreased.

(4) Li, P.; et al. Experimental Study on the Performance of Polyurethane-Steel Sandwich Structure under Debris Flow [4].

To strengthen the impact resistance of buildings subjected to debris flow, this paper proposes the use of a special material, which is namely polyurethane-steel sandwich composite, as the structural material, which generates the polyurethane-steel sandwich structure. The impact resistance of 
polyurethane-steel sandwich structure under debris flow is investigated by a series of impact loading tests, which allows for comparison with the test results of traditional steel frame structures. The test results demonstrate that: (a) the steel frame structure mainly depends on the impacted column to resist the impact loading; and (b) when subjected to debris flow, the polyurethane-steel sandwich structure exhibits superior performance in resisting the impact loading.

(5) Wang, Z.; et al. Development of a Self-Powered Magnetorheological Damper System for Cable Vibration Control [5].

In this paper, a new nonlinear dissipative device, which is the self-powered magnetorheological (MR) damper control system, is applied to attenuate the undesirable vibration of long stay cables. The vibration mitigation performance of the presented self-powered MR damper system is evaluated by model tests with a 21.6-m long cable. The experimental results show that: (a) the supplemental modal damping ratios of the cable in the first four modes can be significantly enhanced by the self-powered MR damper system, demonstrating the feasibility and effectiveness of the new smart passive system; and (b) both the self-powered MR damper and the generator are quite similar to a combination of a traditional linear viscous damper and a negative stiffness device, with the negative stiffness being able to enhance the mitigation efficiency against cable vibration.

Current cutting-edge research. Since it is really common that the vibrating structures would present nonlinear behaviors when being subjected to strong excitations, we occasionally use the nonlinear deformation in the main structure to dissipate vibration energy. Undoubtedly, the nonlinear properties of vibrating structures should be considered when estimating the seismic performance of structures and evaluating the damping performance of dampers. In this sense, some scholars proposed new simulation tools for vibrating structures currently in a nonlinear stage, which complements the current cutting-edge research of the analysis methods for nonlinear vibrating structures. The papers in this category and corresponding comments are listed below:

(6) Chikhaoui, K.; et al. Robustness Analysis of the Collective Nonlinear Dynamics of a Periodic Coupled Pendulums Chain [6].

The paper conducts the robustness analysis of a special nonlinear system, which is namely the periodic coupled pendulums chain, by a generic discrete analytical model. The main contribution of this paper is that the robustness analysis results demonstrate the benefits of the presence of imperfections in such periodic structures. To be more specific, imperfections can be utilized to generate energy localization that is suitable for several engineering applications, such as vibration energy harvesting.

(7) Ye, J.; et al. Member Discrete Element Method for Static and Dynamic Responses Analysis of Steel Frames with Semi-Rigid Joints [7].

This paper's objective is to investigate the complex behaviors of steel frames with nonlinear semi-rigid connections, including both static and dynamic responses, by a simple and effective numerical method that is based on the Member Discrete Element Method (MDEM). The advantages of the proposed simulation approach are as follows: (a) the modified MDEM can accurately capture the linear and nonlinear behavior of semi-rigid connections; and (b) the modified MDEM can avoid the difficulties of finite element method (FEM) in dealing with strong nonlinearity and discontinuity.

(8) Mansouri, I.; et al. Prediction of Ultimate Strain and Strength of FRP-Confined Concrete Cylinders Using Soft Computing Methods [8].

In this paper, the effectiveness of four different soft computing methods for predicting the ultimate strength and strain of concrete cylinders confined with fiber-reinforced polymer (FRP) sheets is evaluated, including radial basis neural network (RBNN), adaptive neuro fuzzy inference system (ANFIS) with subtractive clustering (ANFIS-SC), ANFIS with fuzzy c-means clustering (ANFIS-FCM) 
and M5 model tree (M5Tree). The comparison results show that the ANFIS-SC, performed slightly better than the RBNN and ANFIS-FCM in estimating the ultimate strain of confined concrete. On the other hand, M5Tree provided the most inaccurate strength and strain estimates.

(9) Wen, B.; et al. Soil-Structure-Equipment Interaction and Influence Factors in an Underground Electrical Substation under Seismic Loads [9].

This paper proposes a seismic response analysis method for underground electrical substations considering the soil-structure-equipment interactions, which is performed by changing the earthquake input motions, soil characteristics, electrical equipment type and structure depths. The numerical results indicate that: (a) as a boundary condition of soil-structure, the coupling boundary is feasible in the seismic response of an underground substation; (b) the seismic response of an underground substation is sensitive to burial depth and elastic modulus; (c) the oblique incidence of input motion has a slight influence on the horizontal seismic response, but has a significant impact on the vertical seismic response; and (d) the bottom of the side wall is the seismic weak part of an underground substation, so it is necessary to increase the stiffness of this area.

(10) Liu, C.; et al. Base Pounding Model and Response Analysis of Base-Isolated Structures under Earthquake Excitation [10].

To study the base pounding effects of the base-isolated structure under earthquake excitations, this paper proposes a base pounding theoretical model with a linear spring-gap element. The numerical analysis conducted through this model suggests that: (a) the model offers much flexibility in analyzing base pounding effects; (b) there is a most unfavorable clearance width between adjacent structures; and (c) the structural response increases with pounding and consequently, it is necessary to consider base pounding in the seismic design of base-isolated structures.

(11) Chen, Z.; et al. Application of the Hybrid Simulation Method for the Full-Scale Precast Reinforced Concrete Shear Wall Structure [11].

This paper proposes a new nonlinear seismic performance analysis method for the full-scale precast reinforced concrete shear wall structure based on hybrid simulation (HS). To be more specific, an equivalent force control (EFC) method with an implicit integration algorithm is employed to deal with the numerical integration of the equation of motion (EOM) and the control of the loading device. The accuracy and feasibility of the EFC-based HS method is verified experimentally through the substructure hybrid simulation tests of the pre-cast reinforced concrete shear-wall structure model. Because of the arrangement of the test model, an elastic non-linear numerical model is used to simulate the numerical substructure. The experimental results of the descending stage can be conveniently obtained from the EFC-based HS method.

Industrial application phase. Finally, based on both the theoretical and experimental academic research, the practical designs or optimum methods that are a valuable reference for actual engineering applications can be obtained. In this special issue, one paper proposes a design method for seismically isolated reinforced concrete frame-core tube tall building, while another paper puts forward an optimum method of tuned mass dampers for the pedestrian bridge, both of which are of great industrial interests. The papers in this category and corresponding comments are listed below:

(12) Li, A.; et al. Research on the Rational Yield Ratio of Isolation System and Its Application to the Design of Seismically Isolated Reinforced Concrete Frame-Core Tube Tall Buildings [12].

This paper proposes a high-efficiency design method based on the rational yield ratio of the isolation system and applies it to the design of the seismically isolated reinforced concrete (RC) frame-core tube tall buildings. The main contributions of this paper are as follows. (a) Through 28 carefully designed cases of seismically isolated RC frame-core tube tall buildings, the rational 
yield ratio of the isolation system for such buildings is recommended to be $2-3 \%$. (b) Based on the recommended rational yield ratio, a high-efficiency design method is proposed for seismically isolated $\mathrm{RC}$ frame-core tube tall buildings. (c) The rationality, reliability and efficiency of the proposed method are validated by a case stay of a seismically isolated $R C$ frame-core tube tall building with a height of $84.1 \mathrm{~m}$, which is designed by the proposed design method.

(13) Shi, W.; et al. Application of an Artificial Fish Swarm Algorithm in an Optimum Tuned Mass Damper Design for a Pedestrian Bridge [13].

This paper proposes a new optimization method for the tuned mass damper (TMD), which can be applied to alleviate the vibration of pedestrian bridges based on the artificial fish swarm algorithm (AFSA). The optimization goal of this design method is to minimize the maximum dynamic amplification factor of the primary structure under external harmonic excitations. Through a case study of an optimized TMD based on AFSA for a pedestrian bridge, it was shown that the TMD designed based on AFSA has a smaller maximum dynamic amplification factor than the TMD designed based on other classical optimization methods, while the optimized TMD has a good effect in controlling the human-induced vibrations at different frequencies.

Conflicts of Interest: The authors declare no conflict of interest.

\section{References}

1. Tian, L.; Rong, K.; Zhang, P.; Liu, Y. Vibration control of a power transmission tower with pounding tuned mass damper under multi-component seismic excitations. Appl. Sci. 2017, 7, 477. [CrossRef]

2. Chen, J.; Lu, G.; Li, Y.; Wang, T.; Wang, W.; Song, G. Experimental study on robustness of an eddy current-tuned mass damper. Appl. Sci. 2017, 7, 895. [CrossRef]

3. Wang, W.; Dalton, D.; Hua, X.; Wang, X.; Chen, Z.; Song, G. Experimental study on vibration control of a submerged pipeline model by eddy current tuned mass damper. Appl. Sci. 2017, 7, 987. [CrossRef]

4. Li, P.; Liu, S.; Lu, Z. Experimental study on the performance of polyurethane-steel sandwich structure under debris flow. Appl. Sci. 2017, 7, 1018. [CrossRef]

5. Wang, Z.; Chen, Z.; Gao, H.; Wang, H. Development of a self-powered magnetorheological damper system for cable vibration control. Appl. Sci. 2018, 8, 118. [CrossRef]

6. Chikhaoui, K.; Bitar, D.; Kacem, N.; Bouhaddi, N. Robustness analysis of the collective nonlinear dynamics of a periodic coupled pendulums chain. Appl. Sci. 2017, 7, 684. [CrossRef]

7. Ye, J.; Xu, L. Member discrete element method for static and dynamic responses analysis of steel frames with semi-rigid joints. Appl. Sci. 2017, 7, 714. [CrossRef]

8. Mansouri, I.; Kisi, O.; Sadeghian, P.; Lee, C.-H.; Hu, J. Prediction of ultimate strain and strength of FRP-confined concrete cylinders using soft computing methods. Appl. Sci. 2017, 7, 751. [CrossRef]

9. Wen, B.; Zhang, L.; Niu, D.; Zhang, M. Soil-structure-equipment interaction and influence factors in an underground electrical substation under seismic loads. Appl. Sci. 2017, 7, 1044. [CrossRef]

10. Liu, C.; Yang, W.; Yan, Z.; Lu, Z.; Luo, N. Base pounding model and response analysis of base-isolated structures under earthquake excitation. Appl. Sci. 2017, 7, 1238. [CrossRef]

11. Chen, Z.; Wang, H.; Wang, H.; Jiang, H.; Zhu, X.; Wang, K. Application of the hybrid simulation method for the full-scale precast reinforced concrete shear wall structure. Appl. Sci. 2018, 8, 252. [CrossRef]

12. Li, A.; Yang, C.; Xie, L.; Liu, L.; Zeng, D. Research on the rational yield ratio of isolation system and its application to the design of seismically isolated reinforced concrete frame-core tube tall buildings. Appl. Sci. 2017, 7, 1191. [CrossRef]

13. Shi, W.; Wang, L.; Lu, Z.; Zhang, Q. Application of an artificial fish swarm algorithm in an optimum tuned mass damper design for a pedestrian bridge. Appl. Sci. 2018, 8, 175. [CrossRef] 